\title{
Micronutrient status and associated factors of adiposity in primary school children with normal and high body fat in Colombo municipal area, Sri Lanka
}

Kalaichelvi Thillan ${ }^{1}$, Pulani Lanerolle ${ }^{1}$, Tharanga Thoradeniya ${ }^{1}$, Dulani Samaranayake ${ }^{2}$, Rohana Chandrajith ${ }^{3}$ and Pujitha Wickramasinghe 4* $^{*}$

\begin{abstract}
Background: The prevalence of obesity and associated risk of chronic diseases are increasing among the paediatric population. The effectiveness of preventive measures and interventions are likely to improve when all factors which associate with obesity in a specific target group are considered. Currently such comprehensive data is unavailable for Sri Lankan children aged 8-9 years.

Methods: This paper pertains to the data collected from August-2015 to November-2016 for a case-control study which included cases (high body fat) $(N=160$; males-81) and controls (normal body fat) ( $N=164$; males-80) recruited from primary schools in the Colombo Municipal area. Anthropometry and body composition (Bioelectrical impedance analysis-BIA) were measured. Diet, physical activity and socio-demographic data were collected using validated interviewer administered questionnaires. Serum concentrations of vitamins A, D [25(OH)D], E, folate (serum and red blood cell-RBC), zinc ( $\mathrm{Zn}$ ), selenium (Se), copper (Cu), iron (Fe), magnesium (Mg), calcium (Ca), chromium $(\mathrm{Cr})$, manganese $(\mathrm{Mn})$, cobalt $(\mathrm{Co})$, ferritin, leptin and high sensitivity C-reactive protein (hs-CRP) were assessed using fasting blood samples.
\end{abstract}

Results: Cases were from higher socio-economic strata and spent significantly less time on physical activities, more time on sedentary behaviours and consumed higher energy compared to the controls. Cases from both genders had significantly lower levels of vitamin D $[25(\mathrm{OH}) \mathrm{D}]$, Fe and Mg (all $p<0.05)$ and higher levels of Cu and Ca (all $p<0.01)$ compared to controls. Higher levels of ferritin and $\mathrm{Cr}$ were seen among male $(p<0.001)$ and female $(p>$ $0.05)$ cases compared to the controls. However, total serum folate levels were lower in male $(p<0.01)$ and female $(p>0.05)$ cases while the RBC folate levels were higher among male $(p<0.01)$ and female $(p>0.05)$ cases compared with controls. Vitamins A, E, Se, Mn and Co ( $p>0.05)$ were not significantly different between groups. The inflammatory markers, both hs-CRP and leptin levels were higher among cases $(p<0.001)$ compared to the controls.

(Continued on next page)

* Correspondence: pujithaw@yahoo.com

${ }^{4}$ Department of Paediatrics, Faculty of Medicine, University of Colombo, Kynsey Road, Colombo, Sri Lanka

Full list of author information is available at the end of the article

(c) The Author(s). 2021 Open Access This article is licensed under a Creative Commons Attribution 4.0 International License, which permits use, sharing, adaptation, distribution and reproduction in any medium or format, as long as you give appropriate credit to the original author(s) and the source, provide a link to the Creative Commons licence, and indicate if changes were made. The images or other third party material in this article are included in the article's Creative Commons licence, unless indicated otherwise in a credit line to the material. If material is not included in the article's Creative Commons licence and your intended use is not permitted by statutory regulation or exceeds the permitted use, you will need to obtain permission directly from the copyright holder. To view a copy of this licence, visit http://creativecommons.org/licenses/by/4.0/ The Creative Commons Public Domain Dedication waiver (http://creativecommons.org/publicdomain/zero/1.0/) applies to the data made available in this article, unless otherwise stated in a credit line to the data. 
(Continued from previous page)

Conclusions: This study highlights higher socio-economic status, lower physical activity, more sedentary

behaviours, higher energy intake and inconsistent distribution of micronutrients among the children with high

body fat when compared with the control group. Increased levels of inflammatory markers indicate the presence of the risk of chronic inflammation in children with high body fat.

Keywords: Micronutrient status, Adiposity, Sri Lankan children

\section{Background}

Over the past few decades, the global prevalence of obesity has increased in epidemic proportion, especially among the paediatric population [1]. Based on the WHO's recent estimates, the prevalence of overweight and obesity has increased from $4 \%$ in 1975 to $18 \%$ in 2016 among children and adolescents of 5-19 years, reaching over 340 million (https://www.who.int/newsroom/fact-sheets/detail/obesity-and-overweight). This has led to the increase in prevalence of noncommunicable diseases [2].

Changes in the dietary and physical activity patterns associated with economic development and urbanization known as the nutrition transition, observed in many low and middle income countries [3] has led to the epidemic of overweight and obesity. Obesity and associated chronic diseases are some adverse health outcomes of such nutrition transition [4]. Unhealthy dietary habits initiated during childhood, often continue throughout their life predisposing to chronic diseases in adulthood [5]. Socioeconomic factors such as education level and income of parents, [6] and number of siblings [7] are associated with the prevalence of overweight and obesity and are likely to work through dietary habits. The prevalence of overweight and obesity is increasing among adolescents and young adults in the South Asian region, including Sri Lanka as they experience the nutrient transition due to the rapid economic development and urbanization [8]. Further, overweight and obesity is a significant public health problem among adolescents in Colombo [9]. However, Sri Lankan children have been reported to have a higher percentage of fat mass from their younger age for a given Body Mass Index (BMI) [10].

Based on the epidemiological data, sex-specific differences were witnessed in the prevalence of childhood obesity [11]. The influence of an obesogenic environment on a child, as well as patterns of pubertal fat deposition differ in girls and boys. It is important to recognize the sex difference in micronutrient status and the inflammatory status associated with adiposity and expanding the study by stratifying on gender basis with the population specific body fat cut offs might provide more insights [12-14].
Although there is limited evidence on lifestyle parameters and overweight in children, such studies fail to provide a complete picture on the link between body fat and micronutrient status. Micronutrient deficiency is a major nutrition-related public health issue globally, particularly among children. Vitamin A, zinc, iron and folate deficiencies have been reported among children and adolescents in Sri Lanka [15, 16]. Since micronutrients play an essential role in energy metabolism, adequate intake of micronutrients is necessary for proper metabolism and tissue function [17] However, research into the links between micronutrient status and body fat have yielded conflicting results. Despite positive energy balance, evidence has directed attention towards micronutrient deficiency as a contributory factor to the deposition of fat and the pathogenesis of obesity [18]. Studies have reported a relationship between micronutrient status, mainly deficiencies among obese children [19]. On the other hand, micronutrient supplementation has also been shown to improve body weight in obese individuals [20]. While there is no evidence that explores the associations between micronutrients and body fat among Sri Lankan children, there is also no available data on the status of other important micronutrients such as selenium, copper, magnesium, chromium, manganese, cobalt and vitamin $\mathrm{E}$ in this population.

The role of chronic inflammation in obesity has been widely studied, and a more recent area of interest has been the possibility that low grade chronic inflammation may alter micronutrient status. It has been observed that inflammation reduces the vitamin and mineral levels irrespective of nutrient intake [21]. Hence, low grade chronic inflammation associated with high adiposity could be a contributory factor to micronutrient deficiency. Despite the increasing prevalence of overweight and obesity and concurrent micronutrient deficiencies among the child population in Sri Lanka, such a relationship has not yet been explored.

The risk of developing chronic disease among obese children varies between individuals and it is essential to identify potential risk factors beyond the conventional factors before planning preventive measures or interventions. Often, the failure of preventive measures is due to inadequate understanding of the complexities of the 
undelaying risk factors both biological and environmental. In developing comprehensive preventive strategies, an understanding of associated risk factors of obesity of a specific target group is a prerequisite.

Therefore, the present study aimed to compare micronutrient status, inflammatory markers, physical activity patterns, diet and socioeconomic factors in 8-9 year old children with high and normal body fat living in an urban area of Sri Lanka.

\section{Methods}

\section{Study design, sample and subjects}

This paper pertains to the data collected from August2015 to November-2016 for a case-control study assessing the association between micronutrient status and adiposity carried out in primary school children aged 89 years in an urban area of Sri Lanka. The sample size was initially estimated based on the prevalence data of vitamin $\mathrm{D}$ and iron deficiency among two groups (obese/overweight and normal weight) of children based on published local and regional evidence [22-26]. The highest sample size required per group was 81 , for an estimated prevalence of vitamin D deficiency of 47 and $25 \%$ among obese [23, 24] and normal weight [22] children respectively, an $\alpha$ error of 0.05 , statistical power of $80 \%$ and considering a $10 \%$ dropout rate. Four groups were formed with the stratification by gender in both cases and controls. Further, the sample size adequacy was checked for the analysis of parameters of inflammation (with an $\alpha$ error of 0.05 and the power of $80 \%$ ) using published mean values of leptin [27] among two groups of children.

Cases $(N=160$; male -81$)$ were defined as those having body fat higher than the cut-off value (vide infra) and controls $(N=164$; males-80) were defined as those with normal body fat.

\section{Subject recruitment}

Thirty-seven schools were randomly selected from 110 schools within Colombo Municipal Council area. Overweight/obese and normal weight children were screened based on the BMI (overweight $>+1 \mathrm{SD}$ and $<+2 \mathrm{SD}$ and obese $\geq+2$ SD for each sex, based on BMI for age for 519-year-old children, WHO 2007 standards) during routine school medical inspections which are conducted by Medical Officers of Health of the area in all government and semi- government schools. The school medical inspection carried out among children in grades 1, 4 and 7 , is a routine health screen, identifying problems for treatment or referral and includes the immunization programme. Children in grade 4 (aged 8-9 years) were considered for this study.

Selected children and their parents were invited with the help of their class teacher to the Clinical Laboratory of the Professorial Paediatric Unit of the Lady Ridgeway Hospital which is the main Children's Hospital in Sri Lanka. After obtaining the written consent from parents, cases and controls were recruited according to their body fat measurements. Categorization of cases (male body fat $>28.6 \%$ and female body fat $>33.7 \%$ ) and controls (male body fat $\leq 28.6 \%$ and female body fat $\leq 33.7 \%$ ) were based on the percentage body fat cut-offs for Sri Lankan children [12].

\section{Inclusion and exclusion criteria}

Eight to nine-year old children who were residents of the Colombo Municipal Council area and identified to have normal and high body fat by the cut off value (vide supra) were selected [12].

Children with any chronic illness and on long term medication, children on diet restrictions, birth defects, or congenital anomaly, children on micronutrient supplementation and children with a history of any allergies, infection occurring within 2 weeks of the study were excluded.

Ethical clearance for this study was obtained from the Ethics Review Committees of the Faculty of Medicine, University of Colombo (EC-14-168), and institutional approval from the Lady Ridgeway Children's Hospital, Sri Lanka. Approval was also obtained from the Ministry of Education and the principals of schools to conduct the study. Informed written consent was obtained from parents/guardians of all participants.

\section{Anthropometric and body fat measurements}

Height was measured to the nearest $0.1 \mathrm{~cm}$ without footwear using a potable stadiometer (SECA $225^{\circ}$; Germany), and weight was measured using an electronic weighing scale (SECA $803^{\circ}$; Germany) to the nearest $0.1 \mathrm{~kg}$ with light clothes. BMI was calculated as weight (in kilograms) divided by height squared (in meters). Waist circumference (WC) was measured at the midpoint between the lower border of palpable rib and iliac crest in the mid axillary line, using a non-stretchable tape to the nearest $0.1 \mathrm{~cm}$ and the waist-to-height ratio (WHtR) was calculated as waist circumference (in $\mathrm{cm}$ ) divided by height (in $\mathrm{cm}$ ). Body fat was measured by BIA technique using an In Body $230^{\circ}$ multi-frequency analyzer (Biospace Co., Ltd., Seoul, Korea). All anthropometry measurements were performed according to WHO standards [28] using standardized equipment by a single investigator.

\section{Dietary intake}

An interviewer-based food frequency questionnaire (FFQ) (attached as "Supplementary file 1") was used to collect dietary information from the caregivers of the children. 


\section{Development of FFQ}

The 106 food item FFQ was designed based on the questionnaire used in a previous study in Sri Lanka [29]. The food items were broadly categorized into pulses, rice and rice-based products, wheat flour-based products, breakfast cereals, bakery products, fast food, dairy products, fat/oil, nuts, vegetables/leafy vegetables, fish /meat and meat products/egg, fruits, confectionery, snacks and beverages. The consumption frequency of food item was assessed across the categories "how many times per day", "how many times per week" and "No/rarely/1-2 times per month" with the portion size.

\section{Validation of FFQ}

The contents of the FFQ was evaluated by an expert panel of nutritionists from the Faculty of Medicine University of Colombo, Sri Lanka and the FFQ was modified based on their suggestions.

The validity of the FFQ was assessed against a 7-day food record in a convenient sample of the same study population. Spearman rank correlation coefficient was used to compare the frequency score for the food groups from the FFQ and the 7-day food record. The test-retest reliability (reproducibility) was assessed by administering the questionnaire to a sub-sample of the same study population on two occasions 1 week apart and by calculating the intra-class correlation coefficient (ICC) for frequency scores for the different food groups. The ICC values of $<0.4$ and $\geq 0.76$ were considered as poor and excellent reliability respectively, as reported in the previous study [30].

\section{Assessing the dietary intake}

The validated FFQ was administered to collect dietary information from caregivers by a single investigator. The questionnaire was administered with validated food portion size photographs [31] and commonly used household utensils including cups, bowls with measuring scales and serving spoons to estimate the portion sizes accurately. The amount (portion size in grams) and the frequency of consumption of each food item were assessed per week and total amount consumed per week was estimated by multiplying portion size by consumption frequency per week.

\section{Estimation of nutrient intake}

The nutrient and energy intake was calculated using the food composition tables of the United States Department of Agriculture (USDA) [32], Asian food composition tables [33] and locally published food composition tables [34-36]. The median nutrient intakes were compared with recommended dietary allowances (RDA) for Sri Lankan children aged 6-9 years [37]. Institute of Medicine (IOM) recommendations [38] were used when values were not available for Sri Lankan children. The daily intake of nutrients and energy was calculated as the average from a week's intake.

\section{Identification and exclusion of under reporters of dietary intake}

Under reporters of dietary intake were identified using the formula of Energy intake < Basal metabolic rate $(B M R) \times 1.2$ which can be used as a cut off [39] where the BMR of each subject was calculated using Henry equation [40]. The food and nutrient intakes of children whose daily energy intake (Total energy intake per week/7) was below $[\mathrm{BMR} \times 1.2]$, were excluded from the analysis.

\section{Physical activity}

Physical activity was measured by a physical activity questionnaire (attached as "supplementary file 2"). The questionnaire was adapted from the children physical activity questionnaire (C-PAQ) (https://www.mrcepid.cam.ac.uk/wp-content/uploads/2014/08/CPAQ.

pdf) and modified to accommodate Sri Lankan traditions and culture. Some of the activities in the C-PAQ which were not relevant to Sri Lankan children were substituted with activities that are common. The modified questionnaire was validated using content and criterion validity and test-retest reliability (reproducibility) methods.

Validation of the physical activity questionnaire.

The contents of the physical activity questionnaire were validated, adopting a similar method used in validating the FFQ. The criterion assessment was carried out using ActiGraph wGT3x-BT ${ }^{\bullet}$ triaxial accelerometers https://www.actigraphcorp.com [41] in a different population of children of the same age. The children were instructed to wear the instrument on their right hip during their routine day to day activities except bathing, washing and water sports for seven consecutive days while maintaining a record of worn and removed time of the accelerometer every day. The child's wearing time was defined as a minimum of $480 \mathrm{~min}$ or more on a day. The subjects who had completed at least two weekdays and 1 weekend day were included in the analysis. The average minutes of daily moderate to vigorous physical activity (MVPA) were computed [41]. Age-specific cutoff points were used to determine the minutes of MVPA [42]. The physical activity questionnaire was administered at the end of the week by the investigator. The total time spent on MVPA was calculated. The criterion validity was evaluated by assessing the significant difference between the measurements of mean time spent in MVPA by instrument and the time calculated by the questionnaire. The test-retest reliability was assessed by administering the questionnaire to a sub-sample of the 
same study population on two occasions one week apart with their daily routine activities. The intra-class correlation coefficient (ICC) was used to assess the test-retest reliability of the time spent on each activity, and it was considered acceptable if ICC > $0.7[41,43]$.

\section{Assessment of physical activity}

Physical activity was measured in a typical school week. This questionnaire covered areas related to physical and sedentary activities. The investigator completed the physical activity questionnaire by interviewing the caregivers on the frequency and the total minutes spent on each physical and sedentary activity by the child during the previous week. The activities were categorized based on metabolic equivalent (MET) values published in the Compendium of Physical Activity. Activities which had MET values of $\leq 1.5,3-6$ and $>6$ were classified as sedentary behaviours [44], moderate and vigorous physical activity, respectively [45]. The time spent in both vigorous and moderate physical activities were summed to obtain the total time spent per week in moderate to vigorous physical activities (MVPA) and the average time spent per day was calculated.

\section{Laboratory analysis}

A blood sample $(8 \mathrm{~mL})$ was drawn by an experienced nursing officer using stainless steel needles under standard sterile conditions after a $12 \mathrm{~h}$ overnight fast. Blood samples were collected into covered plain glass tubes without any additives as well as into an EDTA tube for RBC folate analysis. The blood sample collected in the glass tube was centrifuged at $3000 \mathrm{rpm}$ for $10 \mathrm{~min}$ at $4{ }^{\circ} \mathrm{C}$, and serum was separated, aliquoted and stored at $-80^{\circ} \mathrm{C}$ until the analyses were performed.

Serum Vitamin D concentration (25-Hydroxy vitamin D total) was determined using a competitive chemiluminescent immunoassay (Liaison ${ }^{\circ} 25 \mathrm{OH}$ Vitamin D Total, USA). Following an extraction method [46] serum vitamin A (all-trans-retinol) and vitamin $\mathrm{E}$ ( $\alpha$-tocopherol) concentrations were determined simultaneously at the wavelength of $292 \mathrm{~nm}$ by reverse phase (C18 column) HPLC (Shimadzu', Japan) method with an ultraviolet (UV) detector using methanol and water (99:1 ratio) as the mobile phase with retinyl acetate as internal standard. Serum and RBC folate were determined using chemiluminescent assay (ARCHITECT-IP74 ${ }^{\circ}$, USA) according to the manufacturer's instructions.

Serum ferritin was determined using an enzyme link immunosorbent assay (ELISA) (AccuDiag ${ }^{\mathrm{mm}}$, USA) method according to the manufacturer's instructions. $\mathrm{Zn}, \mathrm{Se}, \mathrm{Ca}, \mathrm{Mg}, \mathrm{Cu}, \mathrm{Fe}, \mathrm{Cr}, \mathrm{Mn}$ and $\mathrm{Co}$ were determined using inductively coupled plasma mass spectrometry (ICP-MS) (Thermo ICapQ ${ }^{\circ}$, Fisher Scientific Inc., Bremen, Germany) after samples were digested with trace select nitric acid ( $\geq 69.0 \%$ Trace select; Fluka ${ }^{\circ}$, Germany), hydrochloric acid ( $\geq 37 \%$ Trace select; Fluka ${ }^{\circ}$, Germany) and hydrogen peroxide (35 wt\% Sigma-Aldrich ${ }^{\circ}$, Germany) using microwave digestion system (CEM MARS $6^{\circ}$; USA) equipped with EasyPrep high pressure digestion vessels [47].

hs-CRP and leptin were assessed by a single step immunometric assay (Siemens Healthcare Diagnostics Inc., USA) and an ELISA (EIA-2395 ${ }^{\circ}$, DRG International, Inc., USA) method, respectively.

\section{Quality control}

All samples, where possible, were analyzed in duplicate, with random duplicate samples being analyzed when serum volumes were low. Low and high external quality control sera were used. Pooled serum was used as a bench quality control. The percent coefficient variance was maintained for each assay according to the standard protocol for each assay performed.

\section{Statistical analysis}

Statistical analysis was performed using SPSS for windows version 20 . The analysis was done separately for males and females. Normality of data distribution was assessed by the Kolmogorov-Smirnov test. The mean values of continuous variables were compared using independent sample t-test for variables which were normally distributed. Medians (interquartile range-IQR) were compared using non-parametric Mann -Whitney $\mathrm{U}$ test for the variables which were not normally distributed. The categorical variables were analyzed using chisquare test between cases and controls. Subjects with $\mathrm{CRP}>10 \mathrm{mg} / \mathrm{L}$ were excluded in the comparison of CRP and ferritin between groups. The statistical significant level was set at $p<0.05$.

\section{Results}

Table 1 compares socio demographic, anthropometry and body composition data between case and controls. A higher percentage of cases were from families with a household earning of $L K R \geq 55,000$ (USD $\geq 411.06$ ) per month compared to controls in both sexes. A higher percentage of children in both control groups male and female, had more than one sibling compared to the cases. (The overall data on socio demographic, anthropometry and body composition of cases and controls without stratifying by sex were not shown and included in the Table S1 and attached as ("Additional file 1").

As expected, anthropometry and body composition measures were significantly higher in cases compared to the controls from both genders. The percentage of overweight and obese children assessed by BMI for their age and sex were 63 and $30.8 \%$ among male cases and 72.2 and $25.3 \%$ among female cases, respectively. Others had 
Table 1 Socio demographic, anthropometry and body composition characteristics of cases and controls $N=324$

\begin{tabular}{|c|c|c|c|c|c|c|}
\hline \multirow[t]{2}{*}{ Characteristics } & \multicolumn{3}{|l|}{ Male } & \multicolumn{3}{|l|}{ Female } \\
\hline & $\begin{array}{l}\text { Cases } \\
\% \text { BF > } 28.6 \\
N=81\end{array}$ & $\begin{array}{l}\text { Controls } \\
\% \mathrm{BF} \leq 28.6 \\
N=80\end{array}$ & $p$ value & $\begin{array}{l}\text { Cases } \\
\% \mathrm{BF}>33.7 \\
N=79\end{array}$ & $\begin{array}{l}\text { Controls } \\
\% \mathrm{BF} \leq 33.7 \\
N=84\end{array}$ & $p$ value \\
\hline Age in years & $9.11 \pm 0.323$ & $9.21 \pm 0.356$ & $0.069^{\mathrm{a}^{*}}$ & $9.13 \pm 0.266$ & $9.11 \pm 0.292$ & $0.572^{\mathrm{a}^{* *}}$ \\
\hline \multicolumn{7}{|l|}{ Education level of father $\mathbf{N}(\%)$} \\
\hline Completed primary education & $74(91.4)$ & $56(70.0)$ & $0.001^{a^{*}}$ & $70(88.6)$ & $74(88.1)$ & $0.919^{a^{* *}}$ \\
\hline Not completed & $7(8.6)$ & $24(30.0)$ & & $9(11.4)$ & $10(11.9)$ & \\
\hline \multicolumn{7}{|l|}{ Education level of mother $\mathrm{N}(\%)$} \\
\hline Completed primary education & $75(92.6)$ & $59(73.8)$ & $0.001^{a^{*}}$ & $64(81.0)$ & $74(88.1)$ & $0.210^{\mathrm{a}^{* *}}$ \\
\hline Not completed & $6(7.4)$ & $21(26.2)$ & & $15(19.0)$ & $10(11.9)$ & \\
\hline \multicolumn{7}{|c|}{ Employment status of parents $\mathrm{N}(\%)$} \\
\hline Both employed & $18(22.2)$ & $8(10.0)$ & $0.035^{\mathrm{a}^{*}}$ & $31(39.2)$ & $21(25.0)$ & $0.051^{\mathrm{a}^{* *}}$ \\
\hline One parent employed & $63(77.8)$ & $72(90.0)$ & & $48(60.8)$ & $63(75.0)$ & \\
\hline \multicolumn{7}{|l|}{ Monthly income (LKR) N (\%) } \\
\hline$\geq 55,000.00$ & $46(56.8)$ & $14(17.5)$ & $<0.001^{\mathrm{a}^{*}}$ & $27(34.2)$ & $14(16.7)$ & $0.01^{1^{* *}}$ \\
\hline$<55,000.00$ & $35(43.2)$ & $66(82.5)$ & & $52(65.8)$ & $70(83.3)$ & \\
\hline \multicolumn{7}{|l|}{ Number of siblings $\mathbf{N}(\%)$} \\
\hline$\leq 1$ sibling & $36(44.4)$ & $19(23.8)$ & $0.006^{\mathrm{a}^{*}}$ & $33(41.8)$ & $29(34.5)$ & $0.341^{a^{* *}}$ \\
\hline$>1$ siblings & $45(55.6)$ & $61(76.3)$ & & $46(58.2)$ & $55(65.5)$ & \\
\hline Height (m) & $1.36 \pm 0.06$ & $1.31 \pm 0.07$ & $<0.001^{b^{*}}$ & $1.34 \pm 0.05$ & $1.29 \pm 0.06$ & $<0.001^{b^{* *}}$ \\
\hline Weight (Kg) & $39.46 \pm 1.18$ & $26.12 \pm 1.22$ & $<0.001^{b^{*}}$ & $37.90 \pm 1.16$ & $25.54 \pm 1.19$ & $<0.001^{b^{* *}}$ \\
\hline $\mathrm{BMI}\left(\mathrm{kg} / \mathrm{m}^{2}\right)$ & $20.80(19.1,23.2)$ & $14.64(13.8,17)$ & $<0.001^{c^{*}}$ & $21.02(19.2,22.3)$ & $14.96(13.7,16.3)$ & $<0.001^{c^{* *}}$ \\
\hline \multicolumn{7}{|l|}{ BMI status N (\%) } \\
\hline Normal & $5(6.2)$ & $70(87.5)$ & $<0.001^{\mathrm{a}^{*}}$ & $2(2.5)$ & $78(92.9)$ & $<0.001^{\mathrm{a}^{* *}}$ \\
\hline Overweight & $51(63.0)$ & $10(12.5)$ & & $57(72.2)$ & $6(7.1)$ & \\
\hline Obese & $25(30.8)$ & $0(00.0)$ & & $20(25.3)$ & $0(00.0)$ & \\
\hline$\% \mathrm{BF}$ & $35.8(31.5,40.2)$ & $16.26(12.6,24.1)$ & $<0.001^{c^{*}}$ & $38.4(35.5,42.2)$ & $20.7(15.5,27.1)$ & $<0.001^{c^{* *}}$ \\
\hline $\mathrm{BF}(\mathrm{Kg})$ & $13.8(10.9,17.3)$ & $3.85(2.8,7.3)$ & $<0.001^{c^{*}}$ & $14.6(12.5,16.9)$ & $4.95(3.6,7.5)$ & $<0.001^{\mathrm{c}^{* *}}$ \\
\hline$W C(\mathrm{~cm})$ & $71.09 \pm 1.08$ & $55.37 \pm 1.11$ & $<0.001^{b^{*}}$ & $72.59 \pm 1.12$ & $54.82 \pm 1.12$ & $<0.001^{b^{* *}}$ \\
\hline $\mathrm{WH} \mathrm{tR}$ & $0.53 \pm 1.11$ & $0.42 \pm 1.09$ & $<0.001^{\mathrm{b}^{*}}$ & $0.53 \pm 1.08$ & $0.43 \pm 1.09$ & $<0.001^{b^{* *}}$ \\
\hline
\end{tabular}

$B M I$ Body mass index, WC Waist circumference, WHtR Waist- to- height Ratio, BF Body fat

Monthly income categories were based on the mean household expenditure per month in Sri Lanka-. Household income and expenditure survey- 2016. LKRLankan rupee ( 1 USD $=133.80$ LKR on the day of data collection, 55,000.00 LKR $=411.06$ USD)

"Differences between male cases and controls, ${ }^{* *}$ Differences between female cases and controls, ${ }^{\text {a }}$ Pearson chi-square value, ${ }^{\mathrm{b}}$ Independent sample t-test compared mean $\pm \mathrm{SD}$, ${ }^{C}$ Mann - Whitney $\mathrm{U}$ test compared median (inter quartile range)-statistically significant at $p<0.05$

a normal BMI $(-2 \mathrm{SD}-+1 \mathrm{SD})$. Similarly, $87.5 \%$ of males and $92.9 \%$ of females from the controls group had normal BMI for their age and sex and rest were overweight (Table 1).

Table 2 compares the micronutrient status and inflammatory markers among cases and controls. Serum vitamin D [25 (OH)D] levels were significantly lower among the cases compared to controls. Similarly, the total serum folate levels were lower among cases than controls. However, the RBC folate levels were higher among cases compared to the controls. Serum vitamin $A$ and $E$ levels were not different between the two groups of both sexes.
The serum concentration of $\mathrm{Mg}$ was significantly lower among male $(p<0.01)$ and female $(p<0.001)$ cases compared to controls. Although serum Fe levels were significantly lower in cases, serum ferritin levels were higher than in controls. Ca levels were significantly higher among both male $(p<0.001)$ and female $(p<$ $0.01)$ cases compared to the controls. Similarly, $\mathrm{Cu}$ levels were higher among cases $(p<0.001)$ in both sexes and $\mathrm{Cr}$ among male $(p<0.05)$ cases compared to the controls but higher $\mathrm{Zn}$ levels were observed only in male cases compared to controls. There were no significant differences in the concentrations of Se, Mn and Co between groups in both sexes. Both CRP and leptin levels were 
Table 2 Micronutrient and inflammatory status of cases and controls $N=324$

\begin{tabular}{|c|c|c|c|c|}
\hline Characteristics & Gender & $\begin{array}{l}\text { Cases } \\
\text { Male- } N=81 \% \text { BF }>28.6 \\
\text { Female- } N=79 \% \text { BF }>33.7\end{array}$ & $\begin{array}{l}\text { Controls } \\
\text { Male- } N=\mathbf{8 0} \% \mathrm{BF} \leq \mathbf{2 8 . 6} \\
\text { Female- } N=84 \% \mathrm{BF} \leq \mathbf{3 3 . 7}\end{array}$ & $p$ value \\
\hline \multirow[t]{2}{*}{ Vitamin D (ng/mL) } & Male & $16.19 \pm 5.06$ & $17.72 \pm 4.47$ & $0.045^{\mathrm{a}^{*}}$ \\
\hline & Female & $14.21 \pm 4.13$ & $17.09 \pm 4.93$ & $<0.001^{\mathrm{a}^{* *}}$ \\
\hline \multirow[t]{2}{*}{ Vitamin A ( $\mu \mathrm{g} / \mathrm{dL})$} & Male & $61.94 \pm 16.28$ & $60.72 \pm 17.62$ & $0.647^{\mathrm{a}^{*}}$ \\
\hline & Female & $63.13 \pm 20.29$ & $60.92 \pm 17.41$ & $0.461^{\mathrm{a}^{* *}}$ \\
\hline \multirow[t]{2}{*}{ Vitamin $\mathrm{E}(\mu \mathrm{g} / \mathrm{mL})$} & Male & $4.01(2.9,5.2)$ & $3.64(2.9,5.1)$ & $0.629^{b^{*}}$ \\
\hline & Female & $3.96(2.9,5.4)$ & $3.44(2.2,4.7)$ & $0.057^{b^{* *}}$ \\
\hline \multirow[t]{2}{*}{ Ferritin $(\mathrm{ng} / \mathrm{mL})$} & Male & $36.19(23.6,46.7)$ & $19.3(8.4,29.5)$ & $<0.001^{\mathrm{b}^{*}}$ \\
\hline & Female & $31.76(21.6,40.5)$ & $26.73(16.7,39.5)$ & $0.211^{\mathrm{b}^{* *}}$ \\
\hline Total folate (serum) & Male & $4.58 \pm 1.43$ & $5.36 \pm 1.46$ & $0.007^{\mathrm{a}^{*}}$ \\
\hline (ng/mL) & Female & $4.72 \pm 1.56$ & $5.34 \pm 1.44$ & $0.055^{\mathrm{a}^{* *}}$ \\
\hline Folate (RBC) & Male & $239.01(173.2,274.1)$ & $189.29(163.1,236.9)$ & $0.002^{\mathrm{b}^{*}}$ \\
\hline (ng/mL) & Female & $234.39(171.2,268.6)$ & $208.91(169.4,259.9)$ & $0.303^{b^{* *}}$ \\
\hline \multirow[t]{2}{*}{ Calcium (mg/dL) } & Male & $11.71(10,12.8)$ & $10.58(8.7,11.8)$ & $<0.001^{b^{*}}$ \\
\hline & Female & $11.92(8.6,12.7)$ & $11.23(7.4,12.2)$ & $0.004^{b^{* *}}$ \\
\hline \multirow[t]{2}{*}{ Magnesium (mg/dL) } & Male & $1.70(1.6,1.9)$ & $1.82(1.7,1.9)$ & $0.004^{\mathrm{b}^{*}}$ \\
\hline & Female & $1.68(1.5,1.8)$ & $1.82(1.7,2.0)$ & $<0.001^{b^{* *}}$ \\
\hline \multirow[t]{2}{*}{ Copper $(\mu \mathrm{g} / \mathrm{dL})$} & Male & $142.77(123.3,159.7)$ & $111.56(90.5,135.9)$ & $<0.001^{b^{*}}$ \\
\hline & Female & $137.71(102.5,158.4)$ & $112.87(82.8,133.3)$ & $<0.001^{b^{*+1}}$ \\
\hline \multirow[t]{2}{*}{$\operatorname{Zinc}(\mu \mathrm{g} / \mathrm{dL})$} & Male & $68.78(57.7,83.3)$ & $64.20(51.4,73.8)$ & $0.025^{\mathrm{b}^{*}}$ \\
\hline & Female & $63.02(54.4,72.2)$ & $65.71(45.3,76.2)$ & $0.797^{b^{* *}}$ \\
\hline \multirow[t]{2}{*}{ Selenium ( $\mu \mathrm{g} / \mathrm{dL})$} & Male & $9.51(8.4,10.2)$ & $8.70(7.2,10.5)$ & $0.260^{\mathrm{b}^{*}}$ \\
\hline & Female & $8.86(6.9,10.2)$ & $8.92(7.0,10.2)$ & $0.784^{\mathrm{b}^{* *}}$ \\
\hline \multirow[t]{2}{*}{ Iron $(\mu \mathrm{g} / \mathrm{dL})$} & Male & $61.93(55.2,79.5)$ & $90.84(71.8,105.5)$ & $<0.001^{\mathrm{b}^{*}}$ \\
\hline & Female & $70.24(54.3,86.4)$ & $94.88(79.9,104.8)$ & $<0.001^{\mathrm{b}^{* *}}$ \\
\hline \multirow[t]{2}{*}{ Chromium ( $\mu \mathrm{g} / \mathrm{dL})$} & Male & $0.67(0.32,0.85)$ & $0.21(0.05,0.72)$ & $<0.001^{b^{*}}$ \\
\hline & Female & $0.54(0.14,0.71)$ & $0.33(0.11,0.74)$ & $0.249^{\mathrm{b}^{* *}}$ \\
\hline \multirow[t]{2}{*}{ Manganese ( $\mu \mathrm{g} / \mathrm{dL})$} & Male & $0.28(0.13,0.61)$ & $0.21(0.09,0.45)$ & $0.187^{b^{*}}$ \\
\hline & Female & $0.20(0.11,0.37)$ & $0.27(0.09,0.67)$ & $0.143^{b^{* *}}$ \\
\hline \multirow[t]{2}{*}{ Cobalt ( $\mu \mathrm{g} / \mathrm{dL})$} & Male & $0.018(0.004,0.05)$ & $0.02(0.01,0.04)$ & $0.551^{b^{*}}$ \\
\hline & Female & $0.013(0.004,0.03)$ & $0.02(0.01,0.04)$ & $0.121^{\mathrm{b}^{* *}}$ \\
\hline \multirow[t]{2}{*}{ hs-CRP (mg/L) } & Male & $1.38(0.4,3.4)$ & $0.16(0.08,0.5)$ & $<0.001^{b^{*}}$ \\
\hline & Female & $1.29(0.6,2.6)$ & $0.18(0.05,1.03)$ & $<0.001^{b^{* *}}$ \\
\hline \multirow[t]{2}{*}{ Leptin (ng/mL) } & Male & $9.84(5.5,14.8)$ & $2.19(0.9,5.3)$ & $<0.001^{b^{*}}$ \\
\hline & Female & $15.09(9.7,21.1)$ & $3.71(2.1,6.6)$ & $<0.001^{b^{*-}}$ \\
\hline
\end{tabular}

RBC Red blood cell, $h s$-CRP High Sensitivity C-reactive protein, BF Body fat. The children with hs-CRP level $>10 \mathrm{mg} / \mathrm{L}$ excluded in ferritin and hs-CRP analysis, *Differences between male cases and controls, **Differences between female cases and controls, ${ }^{a}$ Independent sample t-test compared mean \pm SD ${ }^{b}$ MannWhitney $U$ test compared median (inter quartile range), statistically significant at $p<0.05$

significantly higher in cases $(p<0.001)$ compared to the controls in both male and female subjects.

Further analysis on the association of micronutrients and inflammatory markers (data not shown attached as an "Additional file 2"- Table S2), the median levels of hs-CRP and leptin were compared across the lowest and highest tertiles of micronutrients in the combined sample of both cases and controls. A significant reduction of hs-CRP and leptin levels was seen across the lowest and highest tertiles of vitamin D, folate, $\mathrm{Mg}$ and $\mathrm{Fe}$ while an increase was seen across the tertiles of ferritin and $\mathrm{Cu}$. Similarly, a significant rise in hs-CRP and leptin was seen across the tertiles of $\mathrm{Cr}$ and $\mathrm{Ca}$ respectively. '. 


\section{Dietary intake}

Table 3 summarizes the median dietary intake of energy and other nutrients after excluding the under reporters (24 controls and 43 cases where the energy intake was < BMRx1.2). Overall, a higher proportion of under reporters was identified among males $(p<0.05)$ compared to females. Similarly, a higher percentage of under reporters was cases $(p<0.05)$ compared to controls.
However, there was no significant difference in underreporting between cases and controls within the gender groups.

The daily median energy intake of cases was higher compared to that of controls in both sex groups and significant among females. However, carbohydrate was the primary source of energy among all groups of children, and more than $72 \%$ of energy was from carbohydrate.

Table 3 Daily median intake of nutrients of cases and controls $N=257$

\begin{tabular}{|c|c|c|c|c|}
\hline Nutrients intake/day & Gender & $\begin{array}{l}\text { Cases } \\
\mathrm{M}(N=54) / \mathrm{F}(N=63)\end{array}$ & Controls $M(N=63) / F(N=77)$ & $p$ value \\
\hline \multirow[t]{2}{*}{ Energy (Kcal) } & Male & $1908.19(1614.7,2265.9)$ & $1876.58(1526.2,2250.2)$ & $0.299^{*}$ \\
\hline & Female & $2109.10(1805.9,2446.6)$ & $1768.78(1518.4,2318.8)$ & $0.003^{* *}$ \\
\hline \multirow[t]{2}{*}{$\%$ Energy from $\mathrm{CHO}$} & Male & $74.17(70.5,76.7)$ & $73.20(68.3,75.9)$ & $0.137^{*}$ \\
\hline & Female & $74.92(70.9,78.2)$ & $72.74(70.8,77.4)$ & $0.491^{* *}$ \\
\hline \multirow[t]{2}{*}{$\%$ Energy from protein } & Male & $12.15(11.4,13.3)$ & $12.31(11.3,13.4)$ & $0.588^{*}$ \\
\hline & Female & $11.99(11.0,13.0)$ & $12.01(11.1,13.4)$ & $0.588^{* *}$ \\
\hline \multirow[t]{2}{*}{$\%$ Energy from fat } & Male & $12.77(10.5,16.1)$ & $15.13(10.3,17.8)$ & $0.260^{*}$ \\
\hline & Female & $12.61(9.3,16.2)$ & $13.63(9.4,16.1)$ & $0.392^{* *}$ \\
\hline \multirow[t]{2}{*}{ Vitamin A ( $\mu$ g RAE) } & Male & $918.33(531.9,1432.3)$ & $805.80(580.9,1059.1)$ & $0.394^{*}$ \\
\hline & Female & $690.8(418.3,993.1)$ & $778.92(484.2,1059.7)$ & $0.447^{* *}$ \\
\hline \multirow[t]{2}{*}{ Vitamin D (IU) } & Male & $10.33(6.3,19.1)$ & $7.55(3.7,15.8)$ & $0.071^{*}$ \\
\hline & Female & $10.56(5.4,16.0)$ & $9.50(5.1,14.4)$ & $0.253^{* *}$ \\
\hline \multirow[t]{2}{*}{ Vitamin E (mg) } & Male & $4.86(3.2,7.5)$ & $5.18(3.0,7.0)$ & $0.702^{*}$ \\
\hline & Female & $4.24(2.2,6.5)$ & $3.56(2.4,6.2)$ & $0.620^{* *}$ \\
\hline \multirow[t]{2}{*}{ Folate ( $\mu \mathrm{g})$} & Male & $72.04(46.7,123.8)$ & $104.23(59.9,143.9)$ & $0.064^{*}$ \\
\hline & Female & $88.51(54.5,126.7)$ & $96.48(47.5,142.4)$ & $0.637^{* *}$ \\
\hline \multirow[t]{2}{*}{ Zinc (mg) } & Male & $5.40(4.0,6.7)$ & $5.26(4.1,6.3)$ & $0.983^{*}$ \\
\hline & Female & $6.10(4.9,7.6)$ & $5.33(4.0,6.9)$ & $0.097^{* *}$ \\
\hline \multirow[t]{2}{*}{ Selenium ( $\mu \mathrm{g})$} & Male & $50.65(31.5,57.3)$ & $42.28(32.3,55.4)$ & $0.163^{*}$ \\
\hline & Female & $55.82(41.1,73.3)$ & $47.5(36.0,61.0)$ & $0.062^{* *}$ \\
\hline \multirow[t]{2}{*}{ Calcium (mg) } & Male & $432.25(350.5,705.6)$ & $525.81(363.0,780.9)$ & $0.372^{*}$ \\
\hline & Female & $478.07(232.7,763.4)$ & $421.20(293.7,629.2)$ & $0.810^{* *}$ \\
\hline \multirow[t]{2}{*}{ Magnesium (mg) } & Male & $150.93(107.2,225.7)$ & $157.41(99.8,251.1)$ & $0.794^{*}$ \\
\hline & Female & $156.06(100.5,216.1)$ & $148.73(103.6,217.5)$ & $0.870^{* *}$ \\
\hline \multirow[t]{2}{*}{ Iron (mg) } & Male & $14.64(10.1,19.0)$ & $15.36(12.0,19.8)$ & $0.415^{*}$ \\
\hline & Female & $15.42(12.9,18.2)$ & $13.78(11.4,19.0)$ & $0.275^{* *}$ \\
\hline \multirow[t]{2}{*}{ Copper ( $\mu \mathrm{g})$} & Male & $605.04(412.6,840.0)$ & $675.34(415.9,1015.1)$ & $0.455^{*}$ \\
\hline & Female & $601.02(411.7,932.2)$ & $624.32(410.7,980.1)$ & $0.558^{* *}$ \\
\hline \multirow[t]{2}{*}{ Chromium ( $\mu \mathrm{g})$} & Male & $29.72(6.7,51.7)$ & $21.59(3.1,39.0)$ & $0.035^{*}$ \\
\hline & Female & $26.18(7.8,41.2)$ & $21.65(6.8,41.6)$ & $0.781^{* *}$ \\
\hline \multirow[t]{2}{*}{ Manganese (mg) } & Male & $2.54(1.9,3.6)$ & $2.34(1.6,3.4)$ & $0.424^{*}$ \\
\hline & Female & $2.65(2.0,3.4)$ & $2.58(1.7,3.3)$ & $0.447^{* *}$ \\
\hline \multirow[t]{2}{*}{ Cobalt $(\mu g)$} & Male & $2.81(1.9,3.3)$ & $2.42(1.7,3.1)$ & $0.194^{*}$ \\
\hline & Female & $2.61(1.7,3.9)$ & $3.27(2.3,4.2)$ & $0.059^{* *}$ \\
\hline
\end{tabular}


The daily median intake of micronutrients was not significantly different between cases and controls of this study population (Table 3 ).

The daily intake of vitamin D (RDA -600 IU), E (RDA$7 \mathrm{mg}$ ) and Folate (RDA-300 $\mu \mathrm{g}$ ) in both groups were below the recommended levels for this age group (8-9 years) of children except for vitamin A (RDA-500 $\mu \mathrm{g}$ RAE), which was higher than the recommendation.

The intake of Se (RDA-21 mg/day), Mg (RDA-100 mg/ day), $\mathrm{Mn}$ (RDA- $1.5 \mathrm{mg} /$ day), $\mathrm{Cu}(\mathrm{RDA}-440 \mu \mathrm{g} /$ day) and $\mathrm{Cr}(\mathrm{RDA}-15 \mu \mathrm{g} /$ day) were above the recommended levels for both groups while the median intake of Zn (RDA-6 $\mathrm{mg} /$ day), Ca (RDA-700 mg/day) and Fe (RDA-16 mg/ day) were below the recommended level. However, assessment of Co was not possible as there was no RDA available.

\section{Physical activity}

Cases spent less time per day on MVPA compared to the controls in both sex groups (Table 4). Among males, $28.4 \%$ of cases and $76.3 \%$ of controls met the recommendation $(\geq 1 \mathrm{~h} /$ day $)(p<0.001)$ of MVPA whereas among females $44.3 \%$ of cases and $57.1 \%$ of controls met the recommendation.

On average, cases spent more time on screen devices compared to controls in both sexes. However, $61.7 \%$ of male and $39.2 \%$ female cases and $88.8 \%$ male and $85.7 \%$ female controls met the recommendation $(\leq 14 \mathrm{~h}$ per week) of screen activities. Children in the high fat group spent significantly more time on non-screen sedentary activities than their counterparts with normal body fat.

\section{Discussion}

To the best of our knowledge, this is the first study that compares micronutrient status, inflammatory markers, socio-economic factors, diet, physical activity and sedentary behavior patterns related to body fat among 8-9 year old school children in Sri Lanka. This study showed that higher socio-economic status, higher energy intake, lower physical activity, increased levels of inflammatory markers, reduced levels of serum vitamin $\mathrm{D}$, folate, $\mathrm{Fe}$, and $\mathrm{Mg}$ together with increased levels of $\mathrm{RBC}$ folate and serum $\mathrm{Cu}, \mathrm{Ca}$ and $\mathrm{Cr}$ were associated with high body fat.

Table 4 Physical and sedentary activity levels of cases and controls $N=324$

\begin{tabular}{|c|c|c|c|c|}
\hline Characteristics & Gender & $\begin{array}{l}\text { Cases } \\
\text { Males- } N=81 \% B F->28.6 \\
\text { Females- } N=79 \% B F>33.7\end{array}$ & $\begin{array}{l}\text { Control } \\
\text { Males- } N=\mathbf{8 0} \% \mathrm{BF}-\leq \mathbf{2 8 . 6} \\
\text { Females- } N=\mathbf{8 4} \% \mathrm{BF}-\leq \mathbf{3 3 . 7}\end{array}$ & $p$ value \\
\hline \multicolumn{5}{|l|}{ MVPA } \\
\hline Total minutes spent/day & Male & $37.14(8.6,80.4)$ & $95.86(59.9,152.7)$ & $<0.001^{a}$ \\
\hline Median (IQR) & Female & $50.0(15.0,94.3)$ & $72.86(36.1,125.0)$ & $0.005^{\mathrm{a}}$ \\
\hline \multicolumn{5}{|l|}{ Time spent N(\%) } \\
\hline ( $\geq 1 \mathrm{~h} /$ day) & Male & $23(28.4)$ & $61(76.3)$ & $<0.001^{\mathrm{b}}$ \\
\hline (< 1 h/day) & & $58(71.6)$ & $19(23.7)$ & \\
\hline ( $\geq 1$ h/day) & Female & $35(44.3)$ & $48(57.1)$ & $0.101^{b}$ \\
\hline (< 1 h/day) & & $44(55.7)$ & $36(42.9)$ & \\
\hline \multicolumn{5}{|l|}{ Sedentary activities } \\
\hline \multicolumn{5}{|l|}{ Screen activities } \\
\hline Total hours spent/week & Male & $9(4.0,15.5)$ & $7(4,12)$ & $0.074^{\mathrm{a}}$ \\
\hline Median-IQR & Female & $15(5,18)$ & $7(4.2,12)$ & $<0.001^{\mathrm{a}}$ \\
\hline \multicolumn{5}{|l|}{ Time spent N (\%) } \\
\hline$\leq 14 \mathrm{~h} /$ week & Male & $50(61.7)$ & $71(88.8)$ & $<0.001^{\mathrm{a}}$ \\
\hline$>14 \mathrm{~h} /$ week & & $31(38.3)$ & $9(11.2)$ & \\
\hline$\leq 14 \mathrm{~h} /$ week & Female & $31(39.2)$ & $72(85.7)$ & $<0.001^{\mathrm{a}}$ \\
\hline$>14 \mathrm{~h} /$ week & & $48(60.8)$ & $12(14.3)$ & \\
\hline Non- screen activities & Male & $18.0(13.3,26.6)$ & $16.21(11.3,20.6)$ & $0.017^{a}$ \\
\hline $\begin{array}{l}\text { Total hours spent/week } \\
\text { (Median-IQR) }\end{array}$ & Female & $17.92(14,26.8)$ & $15.21(10.8,22.1)$ & $0.017^{\mathrm{a}}$ \\
\hline
\end{tabular}


Higher body fat levels seen in children from a higher socio-economic sector is contrary to the pattern observed in developed countries where the prevalence of obesity was higher in low socio-economic sectors [48]. A similar trend has been reported among primary school children in China [49] and Sri Lankan adults [50] where obesity was seen among higher socio-economic strata. Apart from the fact that people with a high socio economic background have greater access to a variety of food, they may also have more access to computers and smartphones that would promote sedentary behaviour thus promoting weight gain. Further, less number of siblings were seen in the families of cases as reported in other studies [51]. Due to the lack of playmates, their choices of play is likely to be sedentary rather than physical activity.

Higher energy intake is one of the risk factors associated with weight gain. However, under- reporting is a significant problem in evaluating the energy intake in dietary assessments which may influence the estimation of energy intake [52]. A higher percentage of underreporting has been reported in various populations compared to over-reporting. Further, under-reporting has been documented in relation to overweight [53]. Similarly, the prevalence of under-reporting was high among cases in the present study as well. Further, the present study identified a majority of under-reporters among males than females irrespective of body fat.

Thus in the present study, female cases (after excluding under reporters) were found to consume more energy compared to controls. However, the energy intake in all subjects exceeded the recommendation (males$1775 \mathrm{kcal} /$ day, females-1725 kcal/day) [37] for this age group of children in Sri Lanka. Carbohydrate was the primary source of energy in this study population irrespective of body fat. As rice is the staple food in Sri Lanka, the frequency of consumption of rice and ricebased products is high. Besides (data not shown) a higher number of female cases had the habit of snacking mostly on sugared biscuits ( $\geq 2$ times per day) and consuming sweetened carbonated beverages. All these unhealthy dietary habits may contribute to the high energy consumption among female cases.

Further, high sedentary behaviours, low physical activities [54] and higher energy intake of cases may probably associate with the risk of weight gain. On the other hand, a positive relationship has been reported between screen time and unhealthy eating behaviours among Polish adolescents [55]. However, the present study could not explain such relationship; instead, it showed a positive association between unhealthy eating behaviours and adiposity. Perhaps the screen time may directly or indirectly contribute to the risk of weight gain.
The increased levels of hs-CRP (>1 mg/L) [56] and leptin in cases indicated the risk of inflammation. As leptin is secreted by adipose tissue, it increases in proportion to the fat mass. Leptin promotes inflammatory responses by inducing inflammatory cytokine production [57]. Inflammatory cytokines induce the production of CRP in the liver. Further leptin itself has been reported to induce the CRP production in cultured cells [58].

The low vitamin D levels $(<20 \mathrm{ng} / \mathrm{mL})$ observed in both cases and controls is likely due to low dietary intake. The low dietary intake may probably be due to reduced dietary sources in Sri Lanka. The low vitamin D status of cases compared to the controls could be due to the inadequate sunlight exposure as they spent more time indoors with sedentary behaviours than physical activities. Moreover, volumetric dilution of vitamin D within the fat mass reduces its bioavailability as well [59].

Further, the low vitamin D status increases the parathyroid hormone (PTH) level [60]. Besides, a negative correlation between vitamin $\mathrm{D}$ and $\mathrm{PTH}$ as well as the lower mean levels of vitamin D in the high PTH group of Sri Lankan obese children have been reported [61]. Thus the increased PTH level could be the reason for the elevated levels of $\mathrm{Ca}$ among cases than controls. Another possible explanation would be the osteoclastic activity of increased levels of inflammatory cytokines in cases that may increase the Ca levels [62].

The investigators of previous studies have reported lower levels of vitamin A and $\mathrm{E}$ in obese children. Our findings demonstrated a marginal increase of vitamin A (retinol) and $\mathrm{E}$ ( $\alpha$-tocopherol) levels in cases than the controls. However, vitamin $\mathrm{E}$ intake was lower than the recommended level, while vitamin A was above the level in both groups. This probably could be due to the supplementation programmes as well as the emphasis on dietary vitamin A intake. Further, the increased levels of leptin seen in cases have been reported to induce oxidative stress [63]. Vitamins A and E are the fat-soluble antioxidants stored in the adipose tissue. The oxidative stress could be the possible cause for the release of these vitamins from adipose tissue to increase the blood level in cases.

In agreement with our study, low serum folate and increased RBC folate levels in obese individuals despite their low dietary intake of folate have been reported [64]. Serum folate reflects the recent dietary intake. The low serum folate levels in children with high body fat may be due to low dietary intake of folate or the volumetric dilution of folate within the blood. The low serum folate may stimulate the folate uptake by $\mathrm{RBC}$ in the intestine in cases as explained in a previous study [64]. 
Ferritin reflects $\mathrm{Fe}$ stores in the human body and is also an acute-phase protein. Obesity has been reported as an emerging risk of Fe deficiency [65]. The present study showed higher ferritin and lower Fe levels in cases compared to the controls. However, there was no significant difference in Fe intake observed between the two groups. A further potential explanation could be that; the low serum Fe level may be due to decreased Fe absorption by the action of hepcidin which has been reported to increase with inflammation associated with high adiposity [66].

A low level of $\mathrm{Mg}$ was observed in cases despite their higher dietary intake. While a mechanism cannot be suggested, it has been suggested in previous studies that it could be due to either decreased absorption or increased excretion of $\mathrm{Mg}$ in children with higher body fat [67].

In agreement with available data [68], the present study also showed higher levels of $\mathrm{Cu}$ in cases compared to the controls. $\mathrm{Cu}$ has been reported to play an essential role in the regulation of lipid metabolism [69]. Further, $\mathrm{Cu}$ regulates the uptake and utilization of primary metabolic fuels in the adipose tissue through the activity of semucarbazide-sensitive amine oxidase (SSAO). On the other hand, ceruloplasmin $(\mathrm{Cp})$, a cuproprotein serves as a marker of inflammation. The higher levels of $\mathrm{Cu}$ in cases may be likely due to elevated levels these proteins as they require a continuous supply of $\mathrm{Cu}[70]$.

Contradictory to previous data [68] our data showed higher levels of $\mathrm{Zn}$ among male cases compared to controls but no difference was observed among females. There were no significant differences in dietary intake. Similar finding has been reported among obese children in Turkey [71].

Chromium is suggested to be a potentiator of insulin sensitivity [72]. A higher level of $\mathrm{Cr}$ was observed in cases than in controls. This could be due to higher dietary intake but the dietary intake was above the recommended level among cases as well as controls in both genders. The molecular mechanism for increased $\mathrm{Cr}$ level in high adiposity has not been elucidated. However, the documented higher consumption of carbohydrates mainly from rice and rice based products in Sri Lanka could be a contributory factor as rice has been reported to have higher $\mathrm{Cr}$ levels [73].

The present study showed no significant difference in Se levels between cases and controls, as reported in other studies [74]. Few studies have reported low Se levels among obese children compared to non-obese children [68]. Similarly, no difference was observed in $\mathrm{Mn}$ levels between groups. Higher dietary intake of Se and Mn among both cases and control could be the reason for the above findings of the present study. No significant differences were observed in serum Co levels between cases and controls, and we could not compare the serum levels and the dietary intake of Co as there is no recommended dietary intake.

Overall, the present study showed an inconsistent distribution of micronutrients in children with high body fat. Apart from the suggested mechanisms, inflammation could also play a role as it disturbs the micronutrient levels. However, we did not observe any significant change in inflammation in low and high levels of micronutrients among cases; instead, a significant difference was seen in the entire study population.

Although this study confines to a particular population in Sri Lanka with small sample size, it serves as one of the very few studies which described multiple factors associated with adiposity including lifestyle, dietary and biological factors (micronutrients and inflammation) in this age group of children at one glimpse. Further, sexbased description of data was another advantage as sex is an essential factor which influences the body composition as well as the other factors associated with adiposity. Therefore, the data of the present study could be served as preliminary data for future intervention based studies for all races in other countries.

\section{Limitations of the study}

This study mainly focused on an urban paediatric population. This studied population was not a nationally representative sample. Therefore, it could not be generalizable to the entire Sri Lankan paediatric population of this age group. However, it could serve as a representative paediatric population of other urban areas of Sri Lanka where the similar socio-economic background is seen. Further, the sample size was calculated using the available prevalence data of two micronutrients, and the sample size may not have adequate power to detect the association of all micronutrients analyzed with adiposity. Moreover, population specific equations or gold standard method was not used for the identification of under reporters of energy intake. IOM recommended values were used since RDA values for the 8-9 year old Sri Lankan children were not available. However, the RDA values are given for 4-8 and 9-13 year old children and we used the RDA for 4-8 years for the comparison of dietary nutrient intake.

\section{Conclusions}

Contrary to the findings reported in developed countries, the present study showed a significantly higher socioeconomic status among children with high body fat. Children with high body fat (cases) had lower physical activity, higher sedentary behaviours and higher energy intake compared to the controls. The children with high body fat had lower levels of vitamin D, total serum folate, $\mathrm{Fe}, \mathrm{Mg}$ together with higher levels of $\mathrm{RBC}$ folate, $\mathrm{Ca}$, 
$\mathrm{Cu}$ and $\mathrm{Cr}$ compared to the children with normal body fat. The increased levels of hs-CRP and leptin in children with high body fat indicate the risk of underlying chronic inflammation. Further, the significant changes in hs-CRP and leptin levels across the lower and higher levels of micronutrients may indicate the inconsistent distribution of micronutrients observed in the present study. However, future studies are recommended to assess the micronutrient status concerning the inflammation associated with high adiposity.

\section{Supplementary Information}

The online version contains supplementary material available at https://doi. org/10.1186/s12887-020-02473-3.

Additional file 1: Table S1. Socio demographic, anthropometry and body composition characteristics of cases and controls (Sex specific and overall analysis).

Additional file 2: Table S2. hs-CRP and leptin levels across the tertiles of micronutrients in the total study population.

Additional file 3: Supplementary file 1. Food Frequency Questionnaire (FFQ). This FFQ included the food and beverage items commonly consumed by Sri Lankan children. The traditional food items included in this questionnaire were pittu, string hoppers, hoppers, rotti, koththu (made out of rice/wheat flour), fermented food item (made out of black gram and rice), kesel muwa (banana flower), brinjal (eggplant), ambarella (edible fruit with fibrous pit used for cooking) and kola kanda (porridge made out of green leaves, rice and coconut milk)

Additional file 4: Supplementary file 2. Physical activity questionnaire. This questionnaire was the adapted version of children physical activity questionnaire (C-PAQ) (https://www.mrc-epid.cam.ac.uk/ wp-content/uploads/2014/08/CPAQ.pdf.) with culturally sensitive modifications where relevant.

\section{Abbreviations}

RBC: Red blood cell; BMI: Body mass index; BIA: Bioelectrical impedance analysis; WC: Waist circumference; WHtR: Waist-to-height ratio; FFQ: Food frequency questionnaire; C-PAQ: Children physical activity questionnaire; USDA: United States Department of Agriculture; RDA: Recommended dietary allowance; IOM: Institute of Medicine; BMR: Basal metabolic rate; MET: Metabolic equivalent; MVPA: Moderate to vigorous physical activities; EDTA: Ethylenediamine tetraacetic acid; HPLC: High Performance Liquid Chromatography; UV: Ultraviolet; ELISA: Enzyme link immunosorbent assay; ICP-MS: Inductively coupled plasma mass spectrometry; Hs-CRP: High sensitivity C-reactive protein; LKR: Lankan rupee; PTH: Parathyroid hormone; RAE: Retinol activity equivalent; SSAO: Semicarbazide-sensitive amine oxidase; Cp: Ceruloplasmin

\section{Acknowledgements}

We acknowledge Prof. Sumedha Wijeyrathna from Department of Gynaecology and Obstetrics, University of Colombo, the Director, Bandaranayake Memorial Ayurvedic Research Institute Nawinna, Maharagama, Dr. S. Diyabalanage from Department of Geology, Faculty of Science, University of Peradeniya, Mr. T.P. Andrahennadhi for technical support, Department of Biochemistry and Molecular Biology, Faculty of Medicine University of Colombo, Ms. S.M.T.H. Senevirathna, Ms. T.K.G. Rathnayaka, Mr. S.D.D. Dissanayaka, Mr. D.R.S. Jayasinghe from Department of Paediatrics, Faculty of Medicine, University of Colombo, the laboratory technical staff of Vindana Reproductive Center, the staff of Professorial Paediatric Unit at Lady Ridgeway Hospital and the technical staff of Hemas Hospital, Wattala for their support.

We thank all the children who participated in this study and their parents, school principals and class teachers for their support. We also Acknowledge the School Medical Inspection (SMI) team Colombo Municipal Council.

\section{Authors' contributions}

$\mathrm{KT}, \mathrm{PL}, \mathrm{TT}, \mathrm{PW}$ and DS designed the study. KT carried out the study and the laboratory analysis, KT, DS, T, PL and PW analyzed data and wrote the manuscript. RC contributed to the laboratory analysis (ICP-MS) and edited the manuscript. PL and PW edited the manuscript. All authors approved the final version of the paper.

\section{Funding}

This study was funded by the National Science Foundation, Sri Lanka under the competitive research grant scheme. Grant No: RG/2014/HS/09.

\section{Availability of data and materials}

The data set used or analyzed during the current study is not publicly available due to the pending examination of the postgraduate student but are available with the corresponding author on a reasonable request.

\section{Ethics approval and consent to participate}

Ethics approval was obtained from the ethics committees of the Faculty of the Medicine University of Colombo (EC-14-168), Sri Lanka and the Lady Ridgeway Hospital, Sri Lanka. Approvals were obtained from the Ministry of Education and the Principals of Schools. All participants were recruited after obtaining informed written consent from them and their parents.

\section{Consent for publication}

Not applicable.

\section{Competing interests}

The authors declare that they have no competing interests.

\section{Author details}

${ }^{1}$ Department of Biochemistry and Molecular Biology, Faculty of Medicine, University of Colombo, Colombo, Sri Lanka. ${ }^{2}$ Department of Community Medicine, Faculty of Medicine, University of Colombo, Colombo, Sri Lanka. ${ }^{3}$ Department of Geology, Faculty of Science, University of Peradeniya, Peradeniya, Sri Lanka. ${ }^{4}$ Department of Paediatrics, Faculty of Medicine, University of Colombo, Kynsey Road, Colombo, Sri Lanka.

Received: 7 July 2020 Accepted: 15 December 2020

Published online: 06 January 2021

\section{References}

1. de Onis M, Blossner M, Borghi E. Global prevalence and trends of overweight and obesity among preschool children. Am J Clin Nutr. 2010;92: 1257-64. https://doi.org/10.3945/ajcn.2010.29786.

2. Graf C, Ferrar N. Metabolic syndrome in children and adolescents. Review. Visc Med. 2016;32:357-62. https://doi.org/10.1159/000449268.

3. Popkin BM. Nutrition transition and the global diabetes epidemic. Curr Diab Rep. 2015;15(9):64. https://doi.org/10.1007/s11892-015-0631-4.

4. Kac G, Perez-Escamilla R. Nutrition transition and obesity prevention through the life-course. Int J Obes. 2013;3(Suppl):S6-8. https://doi.org/10. 1038/ijosup.2013.3.

5. Saeedi P, Shavandi A, Skidmore PML. What do we know about diet and markers of cardiovascular health in children: a review. Int J Environ Res Public Health. 2019;16(548). https://doi.org/10.3390/ijerph16040548.

6. Ngan HTD, Tuyen LD, Phu PV, Nambiar S. Childhood overweight and obesity amongst primary school children in Hai Phong City, Vietnam. Asia Pac J Clin Nutr. 2018;27(2):399-405. https://doi.org/10.6133/apjcn.062017.08.

7. Park SH, Cormier E. Influence of siblings on child health behaviors and obesity: A systematic review. J Child Fam Stud. 2018;27(7):2069-81. https:// doi.org/10.1007/s10826-018-1049-9.

8. Jayawardena R, Ranasinghe P, Wijayabandara M, Hills AP, Misra A. Nutrition transition and obesity among teenagers and young adults in South Asia. Curr Diabet Rev. 2017;13(5). https://doi.org/10.2174/ 1573399812666160808100211.

9. Hettiarachchi J, Jayatissa R, Wickramasinghe SC, Wijewardena K. Overweight and obesity among adolescent school children in the Colombo education zone. J Postgrad Instit Med. 2018;5(1):E63 1-7. https://doi.org/10.4038/jpgim. 8177.

10. Wickramasinghe VP. Hattori chart based evaluation of body composition and its relation to body mass index in a group of Sri Lankan children. Indian J Pediatr. 2011. https://doi.org/10.1007/s12098-011-0615-6. 
11. Wang VHC, Min J, Xue H, Du S, Xu F, Wang H, Wang Y. Factors contributing to sex differences in childhood obesity prevalence in China. Public Health Nutr. 2018;21(11):2056-64. https://doi.org/10.1017/S1368980018000290.

12. Wickramasinghe VP, Arambepola C, Bandara P, Abeysekera M, Kuruppu S, Dilshan P, Dissanayake BS. Defining obesity using a biological end point in Sri Lankan children. Indian J Pediatr. 2016. https://doi.org/10.1007/s12098016-2191-2.

13. Kirchengast S. Gender differences in body composition from childhood to old age: an evolutionary point of view. J Life Sci. 2010;2(1):1-10. https://doi. org/10.1080/09751270.2010.11885146.

14. Campbell MK. Biological, environmental, and social influences on childhood obesity. Pediatr Res. 2016;79:205-11. https://doi.org/10.1038/pr.2015.208.

15. de Silva A, Atukorala S, Weerasinghe I, Ahluwalia N. Iron supplementation improved iron status and reduced morbidity in children with, or without upper respiratory tract infections: a randomized, controlled study in Colombo, Sri Lanka. Am J Clin Nutr. 2003;77(1):234-41. https://doi.org/10. 1093/ajcn/77.1.234.

16. Thoradeniya T, Atukorala S, Ramanayake R, Wickremasinghe R. Low folic acid status and its association with anaemia in urban adolescent girls and women of childbearing age in Sri Lanka. Br J Nutr. 2006;95(3):511-6. https:// doi.org/10.1079/bjn20051590.

17. Shenkin A. Micronutrients in health and disease. Review. Postgrad Med J. 2006;82(971):559-67. https://doi.org/10.1136/pgmj.2006.047670.

18. Garcia OP, Long KZ, Rosado JL. Impact of micronutrient deficiencies on obesity. Nutr Rev. 2009;67(10):559-72. https://doi.org/10.1111/j.1753-4887. 2009.00228.x.

19. García OP, Ronquillo D, Caamano MDC, Martínez G, Camacho M, Lopez V, Rosado JL. Zinc, iron and vitamins A, C and E are associated with obesity, inflammation, lipid profile and insulin resistance in Mexican school-aged children. Nutrients. 2013;5(12):5012-30. https://doi.org/10.3390/nu5125012.

20. Khosravi ZS, Kafeshani M, Tavasoli P, Zadeh AH, Entezari MH. Effect of vitamin D supplementation on weight loss, glycemic indices, and lipid profile in obese and overweight women: A clinical trial study. Int J Prev Med. 2018;9:63. https://doi.org/10.4103/ijpvm.IJPVM_329_15.

21. Thurnham D, Northrop-Clewes CA. Inflammation and biomarkers of micronutrient status. Curr Opin Clin Nutr Metab Care. 2016;19(6):458-63. https://doi.org/10.1097/MC0.0000000000000323.

22. Hettiarachchi M, Liyanage C. Coexisting micronutrient deficiencies among Sri Lankan pre-school children: a community-based study. J Maternal Child Nutr. 2012;8(2):259-66. https://doi.org/10.1111/j.17408709.2010.00290.x.

23. Peterson CA, Tosh AK, Belenchia AM. Vitamin D in sufficiency and insulin resistance in obese adolescents. Ther Adv Endocrinol Metab. 2014;5(6):16689. https://doi.org/10.1177/2042018814547205.

24. Chung H, Kim JH, Chung S, Yoo E. Vitamin D deficiency in Korean children: prevalence, risk factors, and the relationship with parathyroid hormone levels. Ann Pediatr Endocrinol Metab. 2014;19(2):86-90. https://doi.org/10. 6065/apem.2014.19.2.86.

25. Kapil U, Sareen N. Prevalence of anemia amongst overweight and obese children in NCT of Delhi. Ind J Comm Health. 2014;26(3):295-7 https://www. iapsmupuk.org/journal/index.php/IJCH/article/view/425.

26. Department of Census and Statistics. Demographic \& Health Survey 2006/ 07: Prevalence of Anaemia Among Children and Women in Sri Lanka. 2009. http://www.statistics.gov.lk/Health/Staticallnformation/ PrevalenceOfAnaemiaAmongChildrenANDWomenInSriLanka.

27. Falorni A, Bini V, Molinari D, Papi F, Celi F, Di Stefano G, Berioli MG, Bacosi $\mathrm{ML}$, Contessa G. Leptin serum levels in normal weight and obese children and adolescents: relationship with age, sex, pubertal development, body mass index and insulin. Int J Obes. 1997;21(10):881-90. https://doi.org/10. 1038/sj.ijo.0800485.

28. de Onis M, Onyango AW, den Broeck JV, Chumlea WC, Martorell R, for the WHO Multicentre Growth Reference Study Group. Measurement and standardization protocols for anthropometry used in the construction of a new international growth reference. Food Nutr Bull. 2004;25(suppl 1):S2736. https://doi.org/10.1177/15648265040251S104.

29. de Lanerolle-Dias M, Lanerolle P, Atukorala S, de Silva A. Urbanisation, dietary patterns and body composition changes in adolescent girls: a descriptive cross sectional study. BMC Nutr. 2015;1:30. https://doi.org/10. 1186/s40795-015-0027-5.

30. Chan SG, Ho SC, Kreiger N, Darlington G, Adla EM, So KM, Chong PYY. Validation of a food frequency questionnaire for assessing dietary soy
Isoflavone intake among midlife Chinese women in Hong Kong. J Nutr. 2008;138(3):567-73. https://doi.org/10.1093/jn/138.3.567.

31. Thoradeniya T, de Silva A, Arambepola C, Atukorala S, Lanerolle P. Portion size estimation aids for Asian foods. J Hum Nutr Diet. 2012. https://doi.org/ 10.1111/j.1365-277X.2012.01245.X.

32. USDA Food composition data base. Available at; https://fdc.nal.usda.gov. Accessed 28 Feb 2019

33. Puwastien P, Burlingame B, Raroengwichit M, Sungpuag P. ASEAN Food composition table; 2000. p. 150. www.inmu.mahidol.ac.th/aseanfoods/ composition_data.html.

34. Chandrika UG, Kumara PA. Gotu Kola (Centella asiatica): nutritional properties and plausible health benefits. Adv Food Nutr Res. 2015. https:// doi.org/10.1016/bs.afnr.2015.08.001

35. Nadeeshani H, Wimalasiri KMS, Samarasinghe G, Silva R, Madhujith T. Evaluation of the nutritional value of selected leafy vegetables grown in Sri Lanka. Trop Agric Res. 2018;29(3):255-67. https://doi.org/10.4038/tar.v29i3. 8265.

36. Adikari AM, Thamilini J. Cooking conversion factor of commonly consumed Sri Lankan food items. MOJ Food Process Technol. 2018;6(4):371-4. https:// doi.org/10.15406/mojfpt.2018.06.00190.

37. Recommended dietary allowance for Sri Lankans. Medical Research Institute Colombo. 2007. http://www.mri.gov.lk/assets/Nutrition/2007-RDA-MRI-.pdf. Accessed 3 Mar 2019.

38. Food and Nutrition Board. Institute of Medicine: Dietary reference intakes Recommended dietary allowance. National Academies. https://www. nationalacademies.org/our-work/summary-report-of-the-dietary-referenceintakes. Accessed 12 Dec 2019

39. Schutz Y, Weinsier RL, Hunter GR. Assessment of free-living physical activity in humans: an overview of currently available and proposed new measures. Review. Obes Res. 2001;9(6):368-79. https://doi.org/10.1038/oby.2001.48.

40. Al-Kutbe R, Payne A, de Looy A, Rees GA. A comparison of nutritional intake and daily physical activity of girls aged 8-11 years old in Makkah, Saudi Arabia according to weight status. BMC Public Health. 2017;17:592. https:// doi.org/10.1186/s12889-017-4506-2.

41. Liang Y, Lau PWC, Huang WYJ, Maddison R, Baranowski T. Validity and reliability of questionnaires measuring physical activity self-efficacy, enjoyment, social support among Hong Kong Chinese children. Prev Med Rep. 2014;1:48-52. https://doi.org/10.1016/j.pmedr.2014.09.005.

42. Freedson P, Pober D, Janz KF. Calibration of accelerometer output for children. Med Sci Sports Exerc. 2005;37(11):5523-30. https://doi.org/10.1249/ 01.mss.0000185658.28284.ba.

43. Baumgartner TA, Jackson AS. Measurement for Evaluation in Physical Education and Exercise Science. 6th ed. New York: McGraw-Hill; 1999.

44. Mansoubi M, Pearson N, Clemes SA, Biddle SJH, Bodicoat DH, Tolfrey K, Edwardson $\mathrm{CL}$, Yate $\mathrm{T}$. Energy expenditure during common sitting and standing tasks: examining the 1.5 MET definition of sedentary behavior. BMC Public Health. 2015;15:516. https://doi.org/10.1186/s12889-015-1851-x.

45. General physical activities defined by level of intensity. https://www.cdc.gov/ physicalactivity/downloads/PA_Intensity_table_2_1.pdf. Accessed 8 Nov 2016.

46. Khan A, Khan MI, Iqbal Z, Shah Y, Ahmad L, Watson DG. An optimized and validated RP-HPLC/UV detection method for simultaneous determination of all-trans-retinol (Vitamin A) and á-tocopherol (Vitamin E) in human serum: Comparison of different particulate reversed-phase HPLC columns. J Chromatogr B. 2010;878(25):2339-47. https://doi.org/10.1016/j.jchromb.2010.07.009.

47. Harrington JM, Young DJ, Essader AS, Sumner SJ, Levine KE. Analysis of human serum and whole blood for mineral content by ICP-MS and ICPOES: development of a Mineralomics method. Biol Trace Elem Res. 2014; 160(1):132-42. https://doi.org/10.1007/s12011-014-0033-5.

48. Martinez-Vizcaino V, Solera-Martinez M, Cavero-Redondo I, Garcia-Prieto JC, Arias-Palencia N, Notario-Pacheco B, Martinez-Andres M, Mota J, SanchezLopez M. Association between parental socioeconomic status with underweight and obesity in children from two Spanish birth cohorts: a changing relationship. BMC Public Health. 2015;15:1276. https://doi.org/10. 1186/s12889-015-2569-5.

49. Liu W, Liu W, Lin R, Li B, Pallan M, Cheng KK, Adab P. Socioeconomic determinants of childhood obesity among primary school children in Guangzhou, China. BMC Public Health. 2016;16:482. https://doi.org/10.1186/ s12889-016-3171-1.

50. Katulanda P, Jayawardena MAR, Sheriff MHR, Constantine GR, Matthews DR. Prevalence of overweight and obesity in Sri Lankan adults. Obes Rev. 2010. https://doi.org/10.1111/j.1467-789X.2010.00746.x. 
51. Ochiai H, Shirasawa T, Ohtsu T, Nishimura R, Morimoto A, Obuchi R, Hoshino H, Tajima N, Kokaze A. Number of siblings, birth order, and childhood overweight: a population-based cross-sectional study in Japan. BMC Public Health. 2012;12:766 http://www.biomedcentral.com/1471-245 $8 / 12 / 766$

52. Kye S, Kwon S, Lee S, Lee J, Kim BH, Suh H, Moon H. Under-reporting of energy intake from 24-hour dietary recalls in the Korean National Health and nutrition examination survey. Osong Pub Health Res Perspect. 2014;5(2): 85-91. https://doi.org/10.1016/j.phrp.2014.02.002.

53. Maurer J, Taren DL, Teixeira PJ, Thomson CA, Lohman TG, Going SB, Houtkooper LB. The psychosocial and behavioral characteristics related to energy misreporting. Nutr Rev. 2006;64(2 Pt 1):53-66. https://doi.org/10. 1111/j.1753-4887.2006.tb00188.x.

54. Keane E, Li X, Harrington J, Fitzgerald A. Physical activity, sedentary behavior and the risk of overweight and obesity in school-aged children. Pediatr Exerc Sci. 2017;29(3):408-18. https://doi.org/10.1123/pes.2016-0234.

55. Myszkowska-Ryciak J, Harton A, Lange E, Laskowski W, Wawrzyniak A, Hamulka J, Gajewska D. Reduced Screen time is associated with healthy dietary behaviors but not body weight status among polish adolescents. Report from the Wise Nutrition Healthy Generation Project. Nutrients. 2020; 12(1323). https://doi.org/10.3390/nu12051323.

56. Skinner AC, Steiner MJ, Henderson FW, Perrin EM. Multiple markers of inflammation and weight status: Cross-sectional analyses throughout Childhood. Pediatrics. 2009;125(4). https://doi.org/10.1542/peds.2009-2182.

57. Paz-Filho G, Mastronardi C, Franco CB, Wang KB, Wong M, Licinio J. Leptin: molecular mechanisms, systemic pro-inflammatory effects, and clinical implications. Review. Arq Bras Endocrinol Metab. 2012;56(9):597-607. https:// doi.org/10.1590/s0004-27302012000900001.

58. De Rosa S, Crillo P, Pacileo M, Di Palma V, Paglia A, Chiariello M. Leptin stimulated C-reactive protein production by human coronary artery Endothelial cells. J Vasc Res. 2009;46:609-17. https://doi.org/10.1159/ 000226229.

59. Drincic AT, Armas LAG, Van Diest EE, Heaney RP. Volumetric dilution, rather than sequestration best explains the low vitamin D status of obesity. Obesity. 2012;20(7):1444-8. https://doi.org/10.1038/oby.2011.404.

60. Al-Daghri NM, Yakout S, Aljohani N, Al-Saleh Y, Al-Attas OS, Reginster J, AlDisi D, Alokail MS, Vitamin D. Status and its correlation with parathyroid hormone level among population in Riyadh, Saudi Arabia. J King Saud Univ Sci. 2020;32(3):2016-9. https://doi.org/10.1016/j.jksus.2020.02.002.

61. Adikaram SG, Samaranayake DB, Atapattu N, Kendaragama KM, Senevirathne JT, Wickramasinghe VP. Prevalence of vitamin D deficiency and its association with metabolic derangements among children with obesity. BMC Pediatr. 2019;19:186. https://doi.org/10.1186/s12887-019-1558-8.

62. Souza PP, Lerner UH. The role of cytokines in inflammatory bone loss. Immun Invest. 2013. https://doi.org/10.3109/08820139.2013.822766.

63. Blanca AJ, Ruiz-Armenta MV, Zambrano S, Salsoso R, Miguel-Carrasco JL, Fortuno A, Revilla E, Mate A, Vázquez CM. Leptin induces oxidative stress through activation of NADPH oxidase in renal tubular cells: antioxidant effect of L-Carnitine. J Cell Biochem. 2016;117(10):2281-8. https://doi.org/10. 1002/jcb.25526.

64. Bird JK, Ronnenberg AG, Choi S, Du F, Mason JB, Liu Z. Obesity is associated with increased red blood cell folate despite lower dietary intakes and serum concentrations. J Nutr. 2015;145(1):79-86. https://doi.org/10.3945/jn.114. 199117

65. Aigner E, Feldman A, Datz C. Obesity as an emerging risk factor for Iron deficiency. Nutrients. 2014;6(9):3587-600. https://doi.org/10.3390/nu6093587.

66. Cepeda-Lopez AC, Aeberli I, Zimmermann MB. Does obesity increase risk for iron deficiency? A review of the literature and the potential mechanisms. Int J Vitam Nutr Res. 2010;80(4-5):263-70. https://doi.org/10.1024/0300-9831/ a000033.

67. Hassan SA, Ahmed I, Nasrullah A, Haq S, Ghazanfar H, Sheikh AB, Zafar R, Askar G, Hamid Z, Khushdil A, Khan A. Comparison of serum magnesium levels in overweight and obese children and normal weight children. Cureus. 2017;9(8):e1607. https://doi.org/10.7759/cureus.1607.

68. Azab SFA, Saleh SH, Elsaeed WF, Elshafie MA, Sherief LM, Esh AMH. Serum trace elements in obese Egyptian children: a case control study. Ital J Pediatr. 2014;40:20. https://doi.org/10.1186/1824-7288-40-20.

69. Burkhead $J \mathrm{~L}$, Lutsenko $\mathrm{S}$. The role of copper as a modifier of lipid metabolism. Intech. 2013:40-60. https://doi.org/10.5772/51819.

70. Yang H, Liu CN, Wolf RM, Ralle M, Dev S, Pierson H, Askin F, Steele KE, Magnuson TH, Schweitzer MA, Wong GW. Obesity is associated with copper elevation in serum and tissues. Metallomics. 2019;11(8):1363-71. https://doi. org/10.1039/c9mt00148d.

71. Yakinci C, Paç A, Kuçukbay FZ, Tayfun M, Gul A. Serum zinc, copper, and magnesium levels in obese children. Acta Paediatr Jpn. 1997;39(3):339-41. https://doi.org/10.1111/j.1442-200x.1997.tb03748.x.

72. Hua Y, Clark S, Ren J, Sreejayan N. Molecular mechanisms of chromium in alleviating insulin resistance. J NutrBiochem. 2012;23(4):313-9. https://doi. org/10.1016/j.jnutbio.2011.11.001.

73. Diyabalanage S, Navarathna T, Abeysundara HTK, Rajapakse S, Chandrajith R. Trace elements in native and improved paddy rice from different climatic regions of Sri Lanka: implications for public health. Springer Plus. 2016;5: 1864. https://doi.org/10.1186/s40064-016-3547-9.

74. Bouble DL, Bureau F, Laroche D. Trace element status in obese children: relationship with metabolic risk factors. Eur J Clin Nutr Metabol. 2009;4(2): e98-e100. https://doi.org/10.1016/j.eclnm.2009.01.012.

\section{Publisher's Note}

Springer Nature remains neutral with regard to jurisdictional claims in published maps and institutional affiliations.

Ready to submit your research? Choose BMC and benefit from:

- fast, convenient online submission

- thorough peer review by experienced researchers in your field

- rapid publication on acceptance

- support for research data, including large and complex data types

- gold Open Access which fosters wider collaboration and increased citations

- maximum visibility for your research: over $100 \mathrm{M}$ website views per year

At BMC, research is always in progress.

Learn more biomedcentral.com/submissions 